\title{
Multilayer reconstruction of the $W(001)$ surface
}

\author{
M. S. Altman and P. J. Estrup \\ Department of Physics, Brown University, Providence, Rhode Island 02912
}

I. K. Robinson

AT\&T Bell Laboratories, Murray Hill, New Jersey 07974-2070

(Received 29 April 1988)

\begin{abstract}
$\mathrm{X}$-ray diffraction measurements of the half-order rod intensity profiles arising from the reconstruction of the clean $\mathrm{W}(001)$ surface at low temperature show an unexpected modulation with a period corresponding to the layer spacing. We argue that this can only indicate that the reconstruction involves displacements in at least two layers. The atomic displacements in the second layer are found to be $20 \%$ of those in the top layer.
\end{abstract}

Relaxation and reconstruction are spontaneous modifications of bulk crystal structure that take place at the surface to lower the free energy. The distinction between them concerns the point-group symmetry of the surface. The imposition of a boundary on a crystal limits the possible symmetry elements to those with axes or planes perpendicular to it. Relaxation denotes atomic displacements that conserve all these symmetry elements; it has been observed to involve up to four layers. ${ }^{1}$ Reconstruction, on the other hand, implies spontaneous loss of symmetry from the ideal surface. Once reconstruction is favored in the top layer of a crystal, the atoms of the layers underneath find themselves in environments of lower symmetry and so are no longer constrained to retain high-symmetry sites. Reconstruction is therefore generally expected to propagate into the bulk in much the same way as relaxation. We report here that the $W(001)$ surface has deeper reconstructed layers with displacements at least down to the second layer.

At temperatures below about $250 \mathrm{~K}$, the stable $\mathrm{W}(001)$ surface has a $(\sqrt{2} \times \sqrt{2}) R 45^{\circ}$ structure rather than the $(1 \times 1)$ periodicity observed at room temperature. ${ }^{2,3}$ Extinctions of extra spots in the low-energy electron diffraction (LEED) pattern, ${ }^{3,4}$ observable when surface distortions with one of two equivalent orientations happen to predominate, suggested a structural model with lateral displacements of surface $W$ atoms in the [110] direc tion. This model (Fig. 1) was supported by LEED intensity analyses ${ }^{5,6}$ which gave values for the displacement and interlayer spacing but was disputed by results of high-energy ion scattering measurements. ${ }^{7}$ Theoretical studies $^{8}$ have sought to determine the stabilization energy of the reconstructed phase as well as the driving mechanism of the phase transition from $(1 \times 1)$ to $(\sqrt{2} \times \sqrt{2})$. It has been proposed that the reconstruction involves the softening of surface phonons ${ }^{9}$ and recent helium-atom diffraction measurements appear to support this mechanism. ${ }^{10}$ Here we report new results for the reconstructed W(001) surface obtained by $x$-ray diffraction which answer some of the structural questions unambiguously.

The great advantage of $x$-ray diffraction over LEED for structural determinations is that the intensity of scattered radiation can be analyzed with straightforward kinematical theory: the diffraction pattern is the Fourier transform of the electron density. Previously, surface structural $x$-ray experiments have concentrated on the projection of the structure onto the surface plane and therefore measured the diffraction at perpendicular momentum transfer $l$ close to zero. ${ }^{11,12}$ Although this has some advantages for signal-to-background ratio, $l \simeq 0$ is not a fundamental restriction, and attempts have been made to use a limited range of out-of-plane $(l>0)$ data. ${ }^{12,13}$ In this paper, we examine the variation of the intensity along the half-order rods for W(001). By opting for an unrestricted symmetric four-circle diffraction geometry ${ }^{14}$ we can cover a much wider range of perpendicular momentum transfer (up to $l=1.6$ which is 3.2 $\AA^{-1}$ ) than previously attained ${ }^{12,13}$ and reach regions of reciprocal space previously inaccessible in surface experiments. This advance, combined with the extra care in the data correction described below, were essential to record

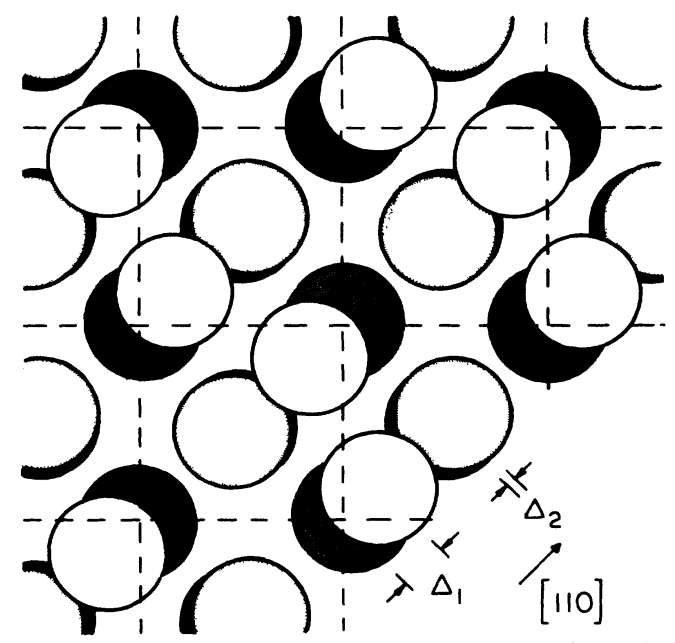

FIG. 1. Top view of the atomic positions in the $\mathbf{W}(001)$ surface. Displacements in the first and second layers from ideal bcc bulk sites are denoted $\Delta_{1}$ and $\Delta_{2}$. This allows the third- and fourth-layer atoms to be seen. Deeper layers are more heavily shaded. Relative displacements are exaggerated three times. Prior to this work only the top-layer displacements were known to exist. 
the intensity modulations reliably. Since measurements were always far from bulk Bragg peaks and the thermal diffuse scatter (TDS) surrounding them, background was not found to be a problem, even at these large incidence angles.

The experiments used $\mathrm{Cu} K \alpha$ x rays $(\lambda=1.542 \AA)$ from a $60-\mathrm{kW}$ rotating-anode source. The entire ultrahigh-vacuum system (Fig. 2) was mounted on a large four-circle diffractometer operating in the symmetric $\omega=0$ mode. ${ }^{14,15}$ Perpendicular momentum transfer settings were achieved by use of the diffractometer $\chi$ angle varying between $0^{\circ}$ and $60^{\circ}$. Changes in total momentum transfer (up to a factor of 3 ) were compensated by corresponding changes in $\theta$ and $2 \theta$. Figure 2 shows the $\mathrm{Be}$ window in the chamber wall that permitted entry and exit of the x-ray beams. The sample was a single-crystal tungsten ribbon of dimensions $0.25 \times 9 \times 30 \mathrm{~mm}^{3}$, oriented and mechanically polished to within $0.5^{\circ}$ of the $(001)$ plane. Sample temperatures between 150 and $2500 \mathrm{~K}$ could be achieved by combining liquid-nitrogen cooling and resistive heating and were measured with a W-5 wt.\% Re/W-26 wt.\% Re thermocouple. Thermal conduction and expansion of the sample over this wide temperature range was allowed for by a molybdenum sample holder which applied a weak spring tension. The sample was cleaned of bulk and surface carbon impurities initially by flashing to $2300 \mathrm{~K}$ and annealing at $1400 \mathrm{~K}$ in $1 \times 10^{-7}$ Torr of oxygen. Diffraction at the half-order positions was attributed to clean surface reconstruction because the intensity was reversibly dependent upon temperature, becoming undetectable above room temperature, ${ }^{2,3}$ unlike diffraction from most impurity-induced structures. The incommensurate structures characteris-

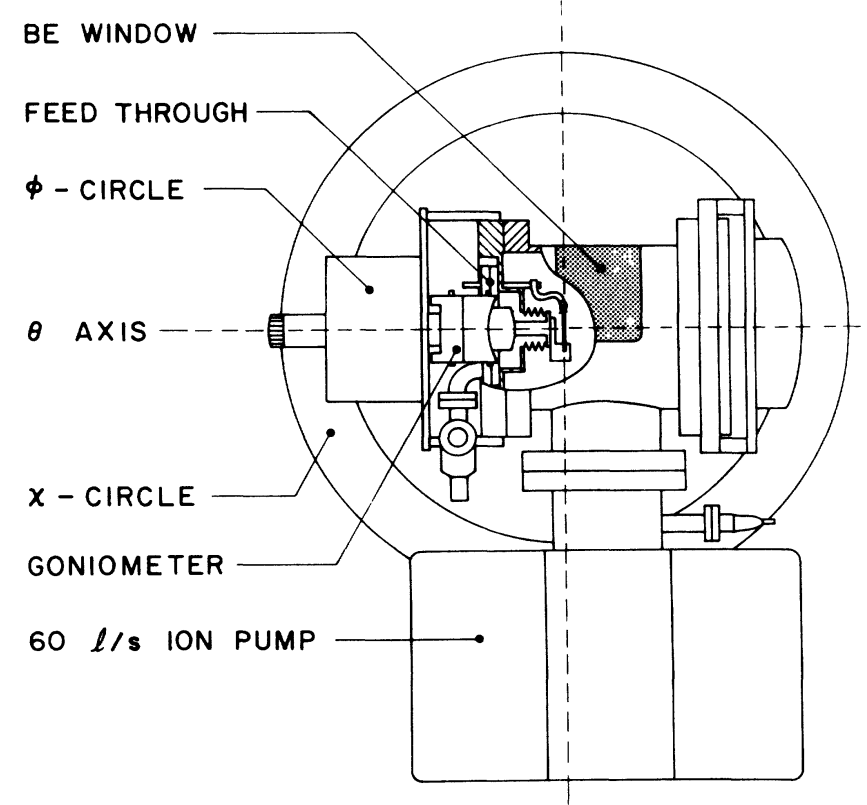

FIG. 2. Experimental apparatus consisting of a portable stainless-steel vacuum chamber mounted on a four-circle $\mathrm{x}$-ray diffractometer. The sample sits at the intersection of the $\theta, \chi$, and $\phi$ axes. Its normal is aligned with the $\phi$ axis by means of a goniometer head, coupled by bellows. $X$ rays enter and leave via a large Be window. tic of hydrogen adsorption ${ }^{16}$ were never observed, indicating negligible $\mathrm{H}_{2}$ in the background gas. The halforder reflections were quenched quickly when the sample was exposed to oxygen, which is known to poison the clean reconstruction. ${ }^{17}$ Following a flash, the extra reflections persisted for an hour with slight intensity decay in a pressure of $10^{-10}$ Torr.

The currently accepted model of the reconstructed, clean $\mathrm{W}(001)$ surface involves displacements of $\mathrm{W}$ atoms exclusively in the top layer (Fig. 1). This makes a simple, testable prediction for $\mathrm{x}$-ray diffraction: the scattered intensity along a reciprocal-lattice rod for a perfect twodimensional system should be constant, except for a gradual decline with $l$ due to the atomic scattering factor and the Debye-Waller factor accounting for thermal motion.

The integrated intensity, $I_{h k}(l)$, was measured at $175 \mathrm{~K}$ along four half-order rods, $\left(\frac{1}{2} \frac{1}{2} l\right),\left(\frac{1}{2} \frac{3}{2} l\right),\left(\frac{3}{2} \frac{3}{2} l\right)$, and $\left(\frac{3}{2} \frac{5}{2} l\right)$.

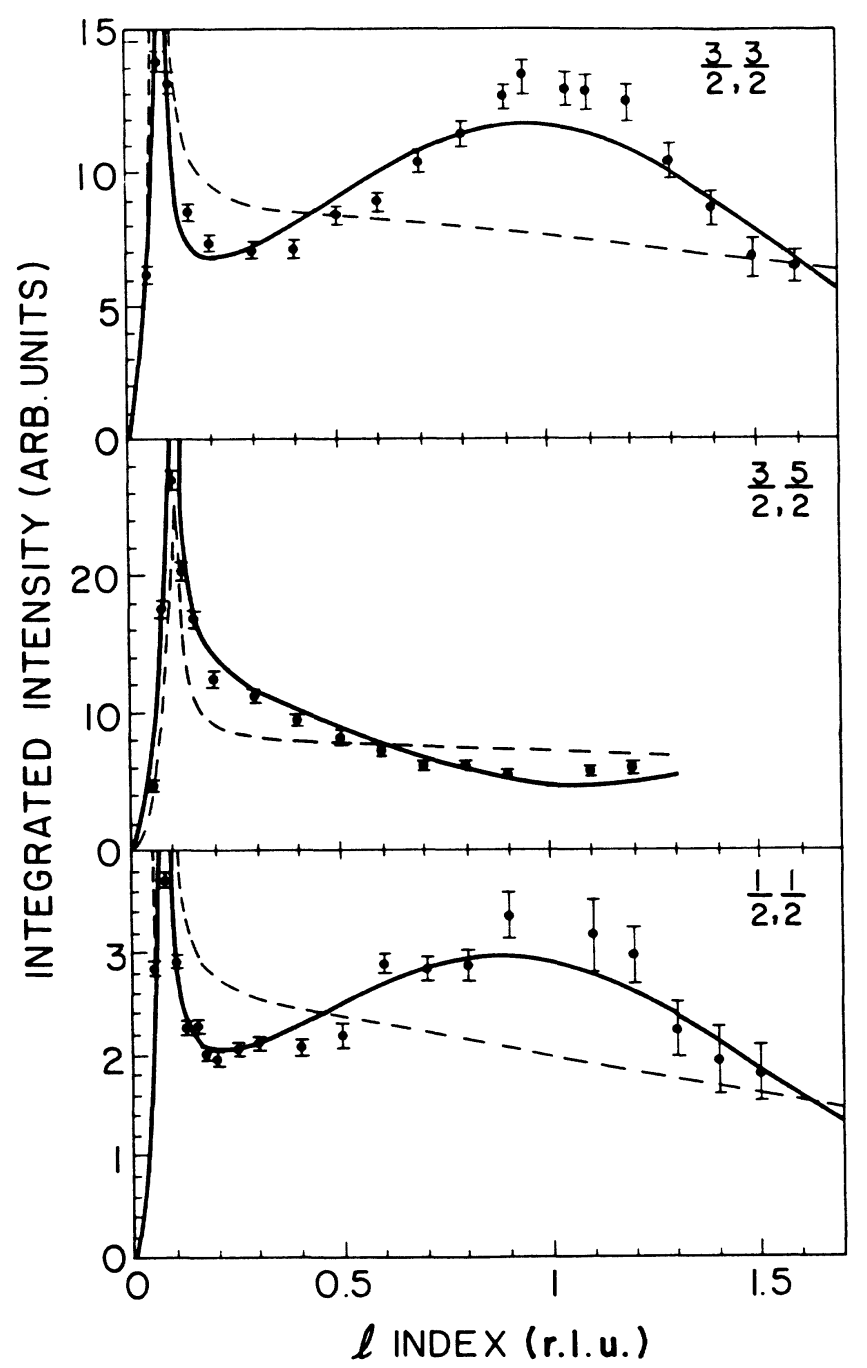

FIG. 3. Measurement of integrated intensity, after correction, along the $\left(\frac{3}{2}, \frac{3}{2}\right),\left(\frac{3}{2}, \frac{5}{2}\right)$, and $\left(\frac{1}{2}, \frac{1}{2}\right)$ rods. The solid curve is the best fit to Eq. (1) with $\Delta_{1}=0.24 \AA, \Delta_{2}=0.046 \AA$, and $d_{12}=1.52 \AA$. The dashed curve corresponds to the one-layer model $\left(\Delta_{1}=0.24 \AA, \Delta_{2}=0\right)$. The perpendicular momentum transfer $l$ is in reciprocal lattice units ( 1 r.l.u. $\left.=2 \pi / a_{0} \AA^{-1}\right)$. 
A striking result, ${ }^{18}$ which contradicts the predictions of the one-layer model, is the pronounced modulation of the intensity along each rod, seen in Fig. 3. Intensities were integrated over azimuthal angle ${ }^{15} \omega$, background subtracted, then corrected for the Lorentz factor $\left[(\sin 2 \theta)^{-1}\right]$, for polarization $\left(1+\cos ^{2} 2 \theta\right)$, and for active area $\left[(\cos \chi \sin 2 \theta)^{-1}\right]$. Furthermore, to correct for resolution effects, the observed intensities were also multiplied by the factor ${ }^{18}$

$$
w_{r}=\left[w_{0}^{2}+\Delta q_{\|}^{2}+\left(\Delta q_{\perp}^{2}-\Delta q_{\|}^{2}\right) \sin ^{2} \chi\right]^{1 / 2}
$$

where $w_{0}$ is the intrinsic half-order rod width, $\Delta q_{\perp}$ is the width of the (Gaussian) resolution function perpendicular to the scattering plane, and $\Delta q_{\|}$is the radial width of the resolution function in the scattering plane. The resolution correction has not been used in previous surface structural work confined to in-plane analysis. It accounts for a progressive loss of integrated intensity (up to 50\%) at larger values of $l$, beyond that required by the $\mathrm{W}$ atomic scattering factor and too large to be realistically described by thermal motion perpendicular to the plane. The radial linewidths were observed to become larger by the same factor and so the effect could be attributed to tilting of the diffraction rods with respect to the instrumental resolution function. In the present case, $\Delta q_{\|}=0.024 \cos (2 \theta / 2) \AA^{-1}$ was known from the resolution limited radial widths of the bulk peaks, and from a fit of $w_{r}$ to the half-order radial linewidths, $w_{0}=0.030$ $\AA^{-1}$ and $\Delta q_{\perp}=0.086 \AA^{-1}$ were then obtained. The sharp maxima at small values of perpendicular momentum transfer are the characteristic peaks due to refraction of the incident and exit beams at the critical angle of total external reflection. ${ }^{19}$

Three descriptions that might explain the oscillatory $l$ dependence of $I_{h k}$ are a buckled surface layer, surface steps, or a multilayer reconstruction. However, we rule out a one-layer buckled model because an unacceptably large buckling amplitude of $a_{0} / 4$ is needed to give the observed period and because it contradicts the observed phases of intensity modulation. Line-shape analysis shows that steps are not the cause of the modulation of the diffracted intensity. If a sufficient density of monoatomic steps were present on the surface that regions of different heights were within the coherence length of the beam, then interference between the regions could produce a modulation. Such effects would also modulate the widths of the diffraction rods ${ }^{20}$ in such a way as to conserve the integrated intensity. We observe no such modulation of the widths either in the radial or the transverse directions. The radial widths were resolvable and gave a domain size of $185 \AA$ (full width at half maximum) after deconvolution.

Both the intensity modulation and the relative intensities of the different orders of diffraction can be explained well by including reconstruction of the second-layer atoms in the same zigzag fashion as the top-layer atoms. The phase of the modulation, which is seen to change between $(h+k)$ even rods and $(h+k)$ odd rods, is consistent with the bcc relationship between the two layers. Furthermore, the observation that $I_{1 / 2,1 / 2}(0)$ and
$I_{3 / 2,3 / 2}(0)$ are minima while $I_{1 / 2,3 / 2}(0)$ and $I_{3 / 2,5 / 2}(0)$ are maxima determines the relative phases of displacements in the two layers: an atom in the top layer and its neighbors in the second layer along the [110] are displaced in the same direction. In the model shown in Fig. 1, $\Delta_{1}$ and $\Delta_{2}$ are the lateral displacements of atoms in the first and second layer, respectively, and $d_{12}$ is their layer separation. The single domain structure factor then has the form

$$
F_{h k}(l)=A_{1}+(-1)^{h+k} A_{2} e^{-2 \pi i l d_{12} / a_{0}}
$$

where

$$
A_{j}=\sin \left\{2 \pi(h+k)\left[(\sqrt{2} / 2) \Delta_{j} / a_{0}\right]\right\}, \quad j=1,2 .
$$

The one-layer model is obtained by $\Delta_{2}=0$.

The intensity data, $I_{h k}(l)$, were fit by the following calculated profile:

$I_{h k}^{\text {calc }}(l)=S|f|^{2}\left|T_{i}\right|^{2} e^{-B\left(h^{2}+k^{2}+l^{2}\right) / 2 a_{0}^{2}}\left|F_{h k}(l)\right|^{2}$

where $S$ is an overall scale factor, $f$ is the atomic scattering factor of $\mathrm{W}, T_{i}$ is the transmission coefficient for the incident beam ${ }^{21}$ describing refraction effects, and the exponential is the Debye-Waller factor. Equal amounts of the two domains, which are related by a rotation of $90^{\circ}$ about the normal, were assumed. From a least-squares analysis, the structural parameters were determined to be $\Delta_{1}=0.24 \pm 0.025 \AA, \Delta_{2}=0.046 \pm 0.016 \AA$, and $d_{12}=1.52 \pm 0.16 \AA$. The interlayer distance is within error of the bulk value. The best-fit value of the DebyeWaller factor yielded $B=0.2 \pm 0.3 \AA^{2}$, corresponding to a vibration amplitude of $0.05 \AA$. This value is comparable with bulk amplitudes (isotropic) in the range 0.025-0.057 $\AA$ A. ${ }^{22}$ Figure 3 shows best-fit curves as well as the prediction from the one-layer model $\left(\Delta_{2}=0\right)$. The discrepancy may indicate reconstruction deeper than the second layer.

Previous determinations of the clean $\mathbf{W}(001)$ $(\sqrt{2} \times \sqrt{2})$ structure by dynamical LEED analyses ${ }^{5,6}$ have not considered reconstruction beyond the first layer. The results, ${ }^{6} \Delta_{1}=0.16 \AA$ and $d_{12}=1.49 \AA$, are in fair agreement with those found by $x$-ray diffraction but it will clearly be of interest to extend the LEED analysis to models with multilayer reconstruction. The interpretation of high-energy ion scattering data $^{7}$ for the disordered $(1 \times 1)$ phase at room temperature may also require revision; the values obtained for the number of displaced atoms and for the magnitude of the displacements will change if it is assumed that distortions in the second layer accompany those in the top layer also above the transition temperature.

While the most detailed total energy calculations for W(001) have obtained values of the displacement, $\Delta_{1}=0.18 \AA$ (Ref. 23 ) and $0.28 \AA,{ }^{24}$ which agree well with our experimental value of $0.24 \AA$, both calculations failed to test for or see second-layer effects. On the other hand, an analysis using a lattice-vibration approach ${ }^{9}$ predicted that distortions penetrate to the second layer and gave a ratio $\Delta_{2} / \Delta_{1}=0.26$, in good agreement with the present data, although the values for the distortions themselves 
were considerably higher than those found here. Recent molecular-dynamics simulations ${ }^{25}$ of $\mathrm{W}(001)$ also predicted second-layer displacements with $\Delta_{2} / \Delta_{1}=0.2$ and $\Delta_{2}=0.05 \AA$. We can say much less about the layer spacing $d_{12}$ because of the large uncertainty in our measurement of a $4 \pm 10 \%$ contraction; however, the existence of the second-layer displacement is bound to affect the value of $d_{12}$ obtained by total energy calculations and may resolve some of the discrepancy between this and the LEED value. ${ }^{6}$ Lastly, the total energy values themselves will be modified when second-layer effects are included, which in turn increases the stability of the reconstruction. This raises the estimates of the transition temperature $T_{c}$ from the reconstructed $(\sqrt{2} \times \sqrt{2})$ to $(1 \times 1)$ phase. ${ }^{26}$
In summary, we have extended previous surface $x$-ray diffraction techniques to obtain a new multilayer picture of the reconstructed $W(001)$ surface. We find that the model based on LEED must be revised to include distortions in the second layer, a result which has significant implications for the interpretation of previous experimental and theoretical studies of this surface.

Note added in proof. C. L. Fu and A. J. Freeman, Phys. Rev. B 37, 2685 (1988), have recalculated the $\mathrm{W}(001)$ energy taking multilayer reconstruction into account. The agreement between the calculated and experimentally determined structure is good.

This work was supported by the National Science Foundation through Grant No. DMR-86-15692.
${ }^{1}$ D. L. Adams and C. S. Sørensen, Surf. Sci. 166, 495 (1986).

${ }^{2}$ T. E. Felter, R. A Barker, and P. J. Estrup, Phys. Rev. Lett. 38, 1158 (1977).

${ }^{3}$ M. Debe and D. King, Phys. Rev. Lett. 39, 708 (1977).

${ }^{4}$ J. C. Wang and T. M. Lu, Surf. Sci. 122, L635 (1982).

${ }^{5}$ R. A. Barker, P. J. Estrup, F. Jona, and P. M. Marcus, Solid State Commun. 25, 375 (1978).

6J. A. Walker, M. K. Debe, and D. A. King, Surf. Sci. 104, 405 (1981).

${ }^{7}$ I. Stensgaard, L. C. Feldman, and P. J. Silverman, Phys. Rev. Lett. 42, 247 (1979).

${ }^{8}$ J. E. Inglesfield, Prog. Surf. Sci. 20, 105 (1985).

${ }^{9}$ A. Fasolino, G. Santoro, and E. Tosatti, Phys. Rev. Lett. 44, 1684 (1980).

${ }^{10}$ H.-J. Ernst, E. Hulpke, and J. P. Toennies, Phys. Rev. Lett. 58, 1941 (1987).

${ }^{11}$ W. C. Marra, P. Eisenberger, and A. Y. Cho, J. Appl. Phys. 50, 6927 (1979); J. Bohr, R. Feidenhans'l, M. Nielsen, M. Toney, R. L. Johnson, and I. K. Robinson, Phys. Rev. Lett. 54, 1275 (1985).

12I. K. Robinson, Phys. Rev. Lett. 50, 1145 (1983).

13J. Skov Pedersen, R. Feidenhans'1, M. Nielsen, K. Kjaer, F. Grey, and R. L. Johnson, Surf. Sci. 189/190, 1047 (1987).

${ }^{14} \mathrm{~W}$. R. Busing and H. A. Levy, Acta Crystallogr. 22, 457 (1967).

${ }^{15}$ Angle conventions for four-circle diffractometry are taken from Ref. 14
${ }^{16}$ R. A. Barker and P. J. Estrup, J. Chem. Phys. 74, 1442 (1981).

${ }^{17}$ L. D. Roelofs, J. W. Chung, S. C. Ying, and P. J. Estrup, Phys. Rev. B 33, 6537 (1986).

${ }^{18}$ I. K. Robinson, M. S. Altman, and P. J. Estrup, Proceedings ICSOS-3 (1987) (Springer, Heidelberg, in press); M. S. Altman, P. J. Estrup, and I. K. Robinson, J. Vac. Sci. Technol. A 6, 630 (1988); I. K. Robinson, Aust. J. Phys. (to be published).

${ }^{19}$ H. Dosch, Phys. Rev. B 35, 2137 (1987); G. Vineyard, ibid. 26, 4146 (1982).

${ }^{20}$ M. Henzler, Appl. Surf. Sci. 11/12, 450 (1982).

${ }^{21}$ From Ref. 19, $T=\left\{2 \sin \alpha /\left[\left(\sin \alpha+c_{1}\right)^{2}+c_{2}^{2}\right]\right\}\left[\left(\sin \alpha+c_{1}\right)\right.$ $\left.+i c_{2}\right] \quad$ with $c_{1,2}=2^{-1 / 2}\left\{ \pm\left(\sin ^{2} \alpha-2 \delta\right)+\left(\sin ^{2} \alpha-2 \delta\right)^{2}\right.$ $\left.\left.+4 \beta^{2}\right]^{1 / 2}\right\}^{1 / 2}$. The variable $\alpha$ is either the incidence or exit angle; the index of refraction is described in terms of $\delta$ and $\beta$ such that $n=1-(\delta+i \beta)$. Only $\left|T_{1}\right|^{2}$ is used instead of $\left|T_{\imath} T_{e}\right|^{2}$ because of degraded resolution of the detected beam perpendicular to the scattering plane.

22 International Tables for X-Ray Crystallography (Reidel, Dordrecht, 1983), Vol. 3.

${ }^{23}$ C. L. Fu, A. J. Freeman, E. Wimmer, and M. Weinert, Phys. Rev. Lett. 54, 2261 (1985).

${ }^{24}$ D. Singh, S. H. Wei, and H. Krakauer, Phys. Rev. Lett. 57, 3292 (1986).

${ }^{25}$ B. Legrand, G. Tréglia, M. C. Desjonquères, and D. Spanjaard, J. Phys. C 19, 4463 (1986).

${ }^{26}$ L. D. Roelofs, Surf. Sci. 178, 396 (1986). 


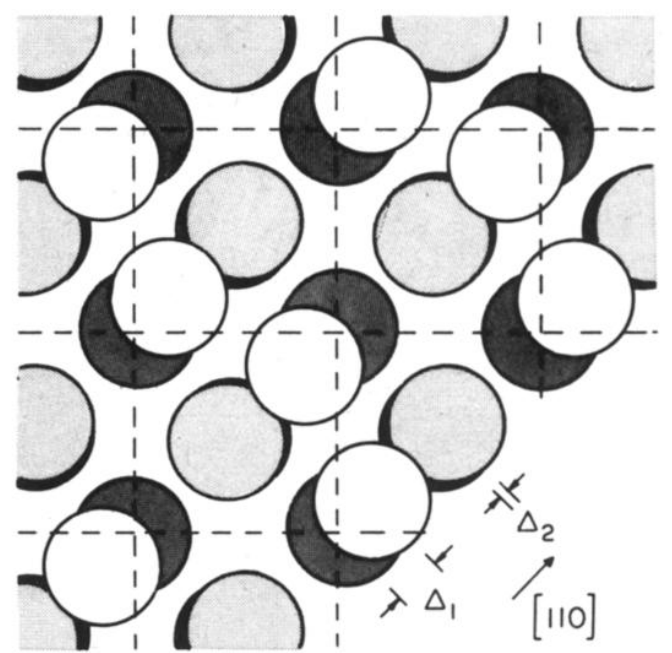

FIG. 1. Top view of the atomic positions in the $\mathrm{W}(001)$ surface. Displacements in the first and second layers from ideal bcc bulk sites are denoted $\Delta_{1}$ and $\Delta_{2}$. This allows the third- and fourth-layer atoms to be seen. Deeper layers are more heavily shaded. Relative displacements are exaggerated three times. Prior to this work only the top-layer displacements were known to exist. 


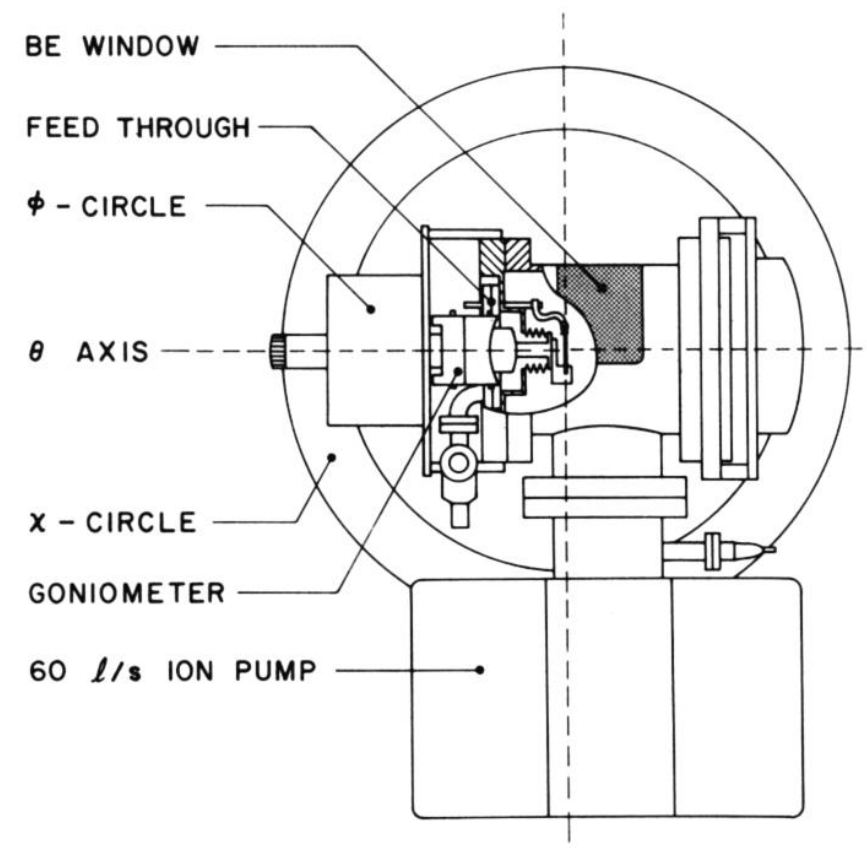

FIG. 2. Experimental apparatus consisting of a portable stainless-steel vacuum chamber mounted on a four-circle $\mathrm{x}$-ray diffractometer. The sample sits at the intersection of the $\theta, \chi$, and $\phi$ axes. Its normal is aligned with the $\phi$ axis by means of a goniometer head, coupled by bellows. $\mathrm{X}$ rays enter and leave via a large Be window. 